\title{
Compressed Sensing to Power Quality Signal with Orthogonal Matching Pursuit Method
}

\author{
OUYANG Hua ${ }^{1, a}$, YANG Zhonglin ${ }^{1, b}$ and LI Hui ${ }^{1, c}$ \\ ${ }^{1}$ Dept. of Electrical Eng. Naval Univ. of Engineering Wuhan 430033, China \\ a78ouyang@163.com, bblueduny@sina.com, ' xybj2008@yeah.net
}

Keywords: Compressed sensing; Power quality; Sparse representation; Orthogonal matching pursuit

\begin{abstract}
Compressed sensing is a new data compression method which can recover a sparse or compressible signal from a small number of linear and non-adaptive measurements. To solve the problems of high sampling rate and massive data storage faced by traditional collection and compression methods of power quality, compressed sensing algorithm is used in the paper. Gaussian random measurement matrix is used to complete the compressed sampling. The orthogonal matching pursuit method is adopted in the reconstruction processes. Simulation show that The SNR of the reconstruction of harmonics signal using compressed sensing is higher than the traditional compression method such as wavelet transform and DCT transform.
\end{abstract}

\section{I . Introduction}

Data acquisition method is one of the key theories and techniques of power quality monitoring system. Traditional methods for acquisition and compression of power quality signals are based on Nyquist sampling theory, which are faced with such troubles as high sampling rate, waste of sampling resources and high attainable cost for hardware. To solve these problems, based on compressed sensing (CS) theory a method for compressed sampling and reconstruction of power quality data is introduced here. Different from the traditional signal acquisition process, compressed sensing, which is a new theory that captures and represents compressible signals at a sampling rate significantly below the Nyquist rate. It first employs non-adaptive linear projections that preserve the structure of the signal, and then the signal reconstruction is conducted using an optimization process from these projections. Compressed sensing has been a research hotspot and a new theoretical framework in the field of signal processing ${ }^{[1-3]}$. Three problems would to be solved: (1) Signal sparse representation. (2) Design of measurement matrix. (3) Reconstruction algorithm matching with sparse decomposition.

\section{Problem formulation}

The basic topic of compressed sensing is to determine the minimal number $n$ of linear nonadaptive measurement that allows for reconstruction of a signal $x \in R^{N}$ that has at most $\mathrm{k}$ nonzero components.

A. Sparse representation. From Fourier transform to wavelet transform, scientists have devoted themselves to find a simple and effective representation of different kind of signal in function space. The object of these transforms is to exploring the sparse of signal, or to enhancing the approximation ability of signal to non-linear function. The theories of compressed sensing think that signal has a very sparse representation in the time domain, or in an orthonormal basis or redundant dictionary, according to that the natural signals and images are significantly sparse in frequency domain. The definition of sparsity is proposed by Donoho in [4]: the transform coefficients of signal $\mathrm{x}$ in orthogonal basis $\{\Psi\}$ is $\Theta=\Psi^{\mathrm{T}} \mathrm{X}$. Let $0<\mathrm{p}<2$ and $\mathrm{R}>0$, if those coefficients satisfy:

$$
\|\Theta\|_{p} \equiv\left(\sum_{i}\left|\theta_{i}\right|^{p}\right)^{1 / p} \leq R
$$


Then we think the coefficients $\Theta$ is sparse in some sense.

The orthonormal basis has been developed for sparse representation early. Because orthonormal transform has the ability of decorrelation and concentration of almost energy in small number of coefficients. This is a rather stringer restriction. Indeed, allowing the signal to be sparse with respect to a redundant dictionary adds a lot of flexibility and significantly extends the range of application. The sparse representation with redundant dictionary was proposed by Mallat and Zhang ${ }^{[5]}$, and the Marching Pursuit (MP) algorithm was proposed, too.

B. Measurement matrix. Measurement matrix $\Phi$ is used When signal is transform to obtain the sparse representation $\Theta=\Psi^{\mathrm{T}} \mathrm{X}$. The work of measurement is to sampling the M observation, where $\mathrm{M}<<\mathrm{N}$, and the signal $\mathrm{x}$ with $\mathrm{N}$ numbers or the equivalent vector $\Theta$ with the basis $\Psi$ can be reconstructive. Obviously if the information is $\mathrm{x}$ is destroyed, the signal recovery would fail. The processing of measurement is to get the projection of sparse coefficients in $M$ row vector of $M \times N$ matrix $\Phi$. In other words, to computer the inner product of the sparse signal with vector in $\Phi$.

$$
Y=\Phi \Theta=\Phi \Psi^{T} \mathbf{X}=A^{C S} \mathbf{X}
$$

Candes and Tao introduce the restricted isometry property (RIP) as a sufficient condition on CS matrix which can ensure the performance of signal recovery ${ }^{[6,7]}$. The property shoes that if $\Phi$ satisfies RIP of order $3 k$ and $\delta_{3 k} \in(0,1)$ :

$$
\left(1-\delta_{3 k}\right)\|z\|_{2}^{2} \leq\left|\Phi_{T} z\right| \leq\left(1+\delta_{3 k}\right)\|z\|_{2}^{2}, \quad \forall z \in R^{|T|}
$$

Where $T \subset\{1,2, \cdots, N\}, \quad|T| \leq 3 k$, the signal $\mathrm{x}$ can be exactly recovered by solving (4)

$$
\min \left\|\Psi^{T} x\right\|_{1} \text {, subject to } \Phi x=y
$$

Many kinds of observation matrix have been explored and investigated; most of them are random matrix including the prominent random Gaussian ensemble; partial Fourier ensemble; partial Hadamard ensemble, and so on.

C. Reconstruction algorithm. A naive approach of recovering $\mathrm{x}$ from $\mathrm{y}$ is solving the $\mathrm{l}_{0}$ minimization problem.

$$
\min \left\|\Psi^{T} \mathbf{X}\right\|_{0} \quad \text { s.t. } \quad A^{C S} \mathbf{X}=\Phi \Psi^{T} \mathbf{X}=\mathbf{Y}
$$

The above approach is not reasonable in practice because its solution in NP-hard ${ }^{[8,9]}$.In order to avoid this severe drawback, there are two kind of methods: one is using greedy algorithms like (orthogonal) matching pursuit (OMP) ${ }^{[5]}$; the other is the basis pursuit(BP) ${ }^{[10]}$.

\section{Simulation test}

Traditional stable power quality signal is harmonics. The basic frequency of simulation signal is $50 \mathrm{~Hz}$, sampling frequency is $12.8 \mathrm{kHz}, 10$ periods of wave are sampled, and the frequency, amplitude and phase of harmonics are showed in table1. The signal cones from the voltage wave of

\begin{tabular}{|c|c|c|c|c|c|c|c|}
\hline $\begin{array}{c}\text { Times of } \\
\text { harmonic }\end{array}$ & 1 & 5 & 7 & 11 & 13 & 17 & 19 \\
\hline Amplitude (\%) & 100 & 35.82 & 10.25 & 7.45 & 3.16 & 3.07 & 1.84 \\
\hline Phase $(0)$ & 0 & 30 & 9.1 & 208.1 & 258.9 & 132.6 & 156.4 \\
\hline
\end{tabular}
three-phase PWM commutator when in uncontrolled rectifier. The amplitude of basis voltage is normalizes to $1 \mathrm{~V}$.

Table 1 composition of harmonic signals

The wave of signal is showed in Fig. 1 (a), and the spectrum is showed in Fig. 1 (b). Obviously the sparsity of signal is $\mathrm{k}=14$ transformed by DFT. 
(a)
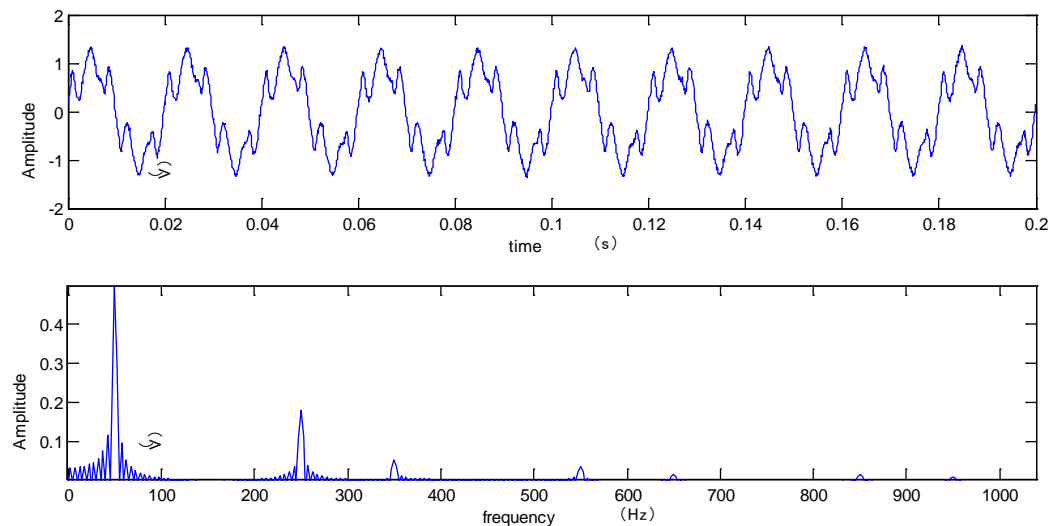

Fig. 1 simulation signal (a) wave of time domain; (b) wave of frequency domain

Comparison of performance between three methods: traditional compression by wavelet and DCT; the compressed sensing in the paper. In consideration the smooth of harmonic signal, the DFT is used for sparse representation. Signal is sampled by Gaussian random measurement matrix. The orthogonal matching pursuit (OMP) method is adopted in the reconstruction processes. SNR of reconstructive signals is showed in table2. It is showed that when compression ratio is high than $4: 1$, the performance of compressed sensing is better than traditional compression method. When compression ratio is high than 8:1, traditional methods are disabled while compressed sensing works well. The original, reconstructive and error signal is showed in Fig. 2.

Table 2 SNR comparison of reconstruction by WT, DCT and CS methods

\begin{tabular}{lcccc}
\hline \multicolumn{1}{c}{$C R$} & $2: 1$ & $4: 1$ & $8: 1$ & $\mathbf{1 6 : 1}$ \\
\hline$S N R(d B)$ & 33.0494 & 30.3307 & 23.6314 & 14.3876 \\
\hline db4 WT & 40.2181 & 35.0716 & 32.0810 & 30.3010 \\
DCT & 39.5217 & 37.5143 & 37.0917 & 32.1551 \\
CS & & &
\end{tabular}

(a)

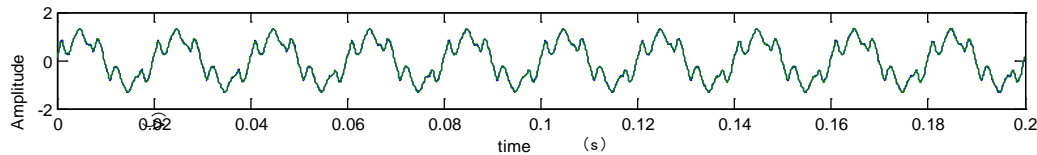

(b)
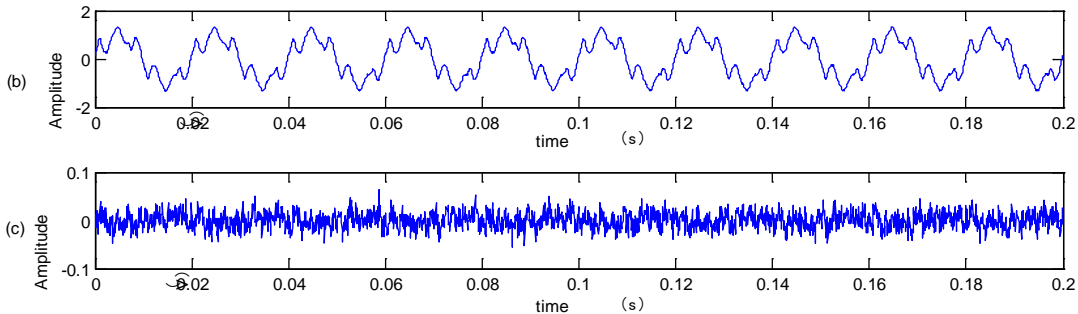

Fig. 2 simulation signal (a) original signal; (b) reconstructive l signal; (c) error signal

\section{Conclusions and future work}

Compressed sensing has been considered as an innovation in signal sampling and acquisition. It has been demonstrated that thin method is better than traditional compressing method. Moreover, there are a number of avenues for further work. DFT sparse representation is useful when only harmonic signal is tested. It would not work well with short-time power quality. Thus redundant dictionary is necessary and effective.

This work was financially supported by the Natural Science Foundation (51307178). 


\section{References}

[1] D.L. Donoho. “Compressed sensing,” IEEE Trans. Inform. Theory, vol.52, no.4, pp. 1289-1306, April 2006.

[2] E. J. Candes, J. K. Romberg, and T. Tao, "Robust uncertainty principle: Exact signal reconstruction from highly incomplete frequency information,” IEEE Trans. Inform. Theory, vol.52, no.2, pp. 489-509, February 2006.

[3]R Baraniuk. A lecture on compressive sensing [J]. IEEE Signal Processing Magazine, 2007, 24 (4) :118-121.

[4] D L Donoho, X Huo. “Uncertainty principles and ideal atomic decompositions”. IEEE Trans. Inform. Theory, 2001, 47 (7): 2845-2862.

[5] S Mallat, Z Zhang. "Matching pursuits with time-frequency dictionaries". IEEE Trans Signal Process, 1993, 41 (12):3397-3415.

[6] E. J. Candes and T. Tao, "Near optimal signal recovery for random projections: Universal encoding strategies?” IEEE Trans. Inform. Theory, vol.52, no.12, pp. 5406-5425, December 2006.

[7]I Daubechies ,M Defrise , C De Mol . “An iterative thresholding algorithm for linear inverse problems with a sparsity constraint”. Comm Pure Appl Math, 2004, 57 (11):1413 - 1457.

[8] M Elad, A M Bruckstein. “A generalized uncertainty principle and sparse representation in pairs of bases”. IEEE Trans. Inform. Theory ,48 (9) :2558-2567

[9] M A T Figueiredo,R D Nowak ,S J Wright . "Gradient projection for sparse reconstruction: Application to compressed sensing and other inverse problems”. IEEE J Selected Topics in Signal Processing: Special Issue on Convex Optimization Methods for Signal Processing, 2007, 1 (4):586 598.

[10] D L Donoho, and M. Saunders, “Atomic decomposition by basis pursuit,”SIAM J. Sci. Comput., vol. 20, no.1, pp. 33-61, 1999. 